\title{
The conjugacy of Collet-Eckmann's map of the interval with the tent map is Holder continuous
}

\author{
T. NOWICKI \\ Department of Mathematics, Warsaw University, \\ 0091 Warsaw, Poland \\ F. PRZYTYCK I \\ Mathematics Institute, Polish Academy of Sciences, Sniadeckich 8, \\ 00-950 Warsaw, Poland
}

(Received 12 June 1987 and revised 10 October 1987)

\begin{abstract}
It is proved that a homeomorphism $h$, which conjugates a smooth unimodal map of the interval with negative Schwarzian derivative and positive Lyapunov exponent along the forward trajectory of the critical value with a tent map, and its inverse $h^{-1}$ are Hölder continuous.
\end{abstract}

\section{Introduction}

We shall deal with a nondegenerate $S$-unimodal map $f$ of the closed interval $\langle 0,1\rangle$ into itself, more exactly we assume that:

(a) $f \in C^{3}, f(0)=f(1)=0$.

(b) There is a unique $c \in(0,1)$ such that $f$ is strictly increasing on $\langle 0, c\rangle$ and strictly decreasing on $\langle c, 1\rangle$ and $f^{\prime \prime}(c) \neq 0$.

(c) $S f \leq 0$ on $\langle 0,1\rangle \backslash\{c\}$ where $S f=f^{\prime \prime \prime} / f^{\prime}-\frac{3}{2}\left(f^{\prime \prime} / f^{\prime}\right)^{2}$.

We use the following notation: $f^{1}=f$ and $f^{n+1}=f^{n} \circ f ; x_{n}=f^{n}(x) ; D f^{n}=$ $(d / d x)\left(f^{n}\right)$. We assume additionally

(d) There are no sinks i.e. for every $p$ with $p_{n}=p,\left|D f^{n}(p)\right|>1$.

Let $(x, y)$ denote the open interval with end points $x$ and $y$ (for $x<y$ as well as $y<x) .|A|$ will denote the Lebesgue measure of the set $A \subset\langle 0,1\rangle$.

Definition 1. We say that $(c, \alpha) \in *(n)$ if $\alpha_{n}=c$ and $\left.D f^{n}\right|_{(c, \alpha)} \neq 0$. Let $(c, \beta)$ be a maximal interval with $(c, \alpha) \subset(c, \beta)$ and $\left.D f^{n}\right|_{(c, \beta)} \neq 0$. We call $\beta$ associated with $\alpha$.

In the sequel we make the following

General assumption. There exist $K>0$ and $\lambda>1$ such that for every $n$ if $(c, \alpha) \in *(n)$ and $\beta$ is associated with $\alpha$ then

$$
\frac{\left|f^{n}(c, \alpha)\right|}{|(c, \alpha)|}>K \lambda^{n} \text { and } \frac{\left|f^{n}(\alpha, \beta)\right|}{|(\alpha, \beta)|}>K \lambda^{n} \text {. }
$$


This assumption is satisfied for example by mappings studied in [CE1, CE2, M, C]. From [N2, N3] it follows that (G.A.) holds if Collet-Eckmann's condition

$$
\liminf _{n \rightarrow \infty} \frac{1}{n} \log \left|D f^{n}\left(c_{1}\right)\right|>0
$$

is satisfied.

Definition 2. Let $g:\langle 0,1\rangle \rightarrow\langle 0,1\rangle$ and $g(x)=\beta / 2-\beta\left|x-\frac{1}{2}\right|$. We call such a map a $\beta$-tent map and denote it by $g_{\beta}$.

It is known ([G] Theorem 4.5, [CE]) that if a map $f$ satisfies (a)-(d) and has no restrictive central point for $n>1$ (the definition will be recalled in $\S 1$ ) then there exists $\beta \in(\sqrt{2}, 2)$ such that $\left.f\right|_{\left(c_{2}, c_{1}\right)}$ is topologically equivalent to $\left.g_{\beta}\right|_{\left(g^{2}\left(\frac{1}{2}\right), g\left(\frac{1}{2}\right)\right)}$, i.e. there exists a homeomorphism $h=h_{f}$ such that $h \circ f=g_{\beta} \circ h$.

The main result of this paper is the following.

ThEOREM. Suppose that $f$ satisfies (a)-(d) and (G.A.) and $f^{n}$ has no restrictive central point for $n>1$. Let $h=h_{f}$ be the homeomorphism conjugating $f$ with a $\beta$-tent map. Then $h$ and $h^{-1}$ are Hölder continuous.

The nontrivial part of this Theorem relies on the following.

MaIn Lemma. For $f$ as in Theorem there exists a constant $\kappa>0$ such that for every interval $J \subset\langle 0,1\rangle$ there exists $m<\kappa \cdot \log (1 /|J|)+1$ which satisfies

$$
f^{m}(J) \supset\left(c_{2}, c_{1}\right) \text {. }
$$

The number 1 is added to $\kappa \cdot \log 1 /|J|$ above to avoid nonsense for $J$ long (of order 1). In the sequel we shall understand that $|J|$ in that kind of estimates is small.

\section{Preliminaries}

We quote some useful properties of maps satisfying (a)-(d) and (G.A.).

$P 1$. ([N2, Lemma 7].) If $\left.D f^{n}\right|_{(a, b)} \neq 0$ then for every $t, x \in(a, b)$

$$
\frac{\left|x_{n}-t_{n}\right|}{|x-t|} \geq \min \left(\frac{x_{n}-a_{n} \mid}{|x-a|}, \frac{\left|x_{n}-b_{n}\right|}{|x-b|}\right) \text {. }
$$

In particular if $t=x$

$$
\left|D f^{n}(x)\right| \geq \min \left(\frac{\left|x_{n}-a_{n}\right|}{|x-a|}, \frac{\left|x_{n}-b_{n}\right|}{|x-b|}\right) .
$$

Definition 3. For $x \in\langle 0,1\rangle$ define $\hat{x}$ by: $\hat{c}=c$ and if $x \neq c$ then $\hat{x} \neq x$ and $f(\hat{x})=f(x)$.

LEMMA 1. If $(c, \alpha) \in *(n)$ with associated $\beta$, then $(c, \hat{\alpha}) \in *(n)$ and $\hat{\beta}$ is associated with $\hat{\alpha}$.

Proof. We have $f^{n}(\hat{\alpha})=f^{n}(\alpha)=c$ so it is enough to prove that $D f^{n}(x)=0$ if and only if $D f^{n}(\hat{x})=0$.

$$
D f^{n}(x)=\prod_{s=0}^{n-1} f^{\prime}\left(x_{s}\right)=\prod_{s=1}^{n-1} f^{\prime}\left(x_{s}\right) \cdot f^{\prime}(x)
$$


As $f^{s}(x)=f^{s}(\hat{x})$ for $s \geq 1$ we see that all factors but the last are equal in $D f^{n}(x)$ and $D f^{n}(\hat{x})$. But $f^{\prime}(x)=0$ or $f^{\prime}(\hat{x})=0$ implies $x=c=\hat{x}$.

$P 2$. ([CE1, Lemma 2.2].) There are two positive numbers $m, M$ such that $m|x-c| \leq$ $\left|f^{\prime}(x)\right| \leq M|x-c|$ (which follows from (a) and (b)).

P3. ([N1, Lemma 3.4].) If $x \neq c$, then $|x-c| /|\hat{x}-c| \leq \sqrt{M} / \sqrt{m}$.

From (G.A.) it follows immediately

LEMMA 2. There exists $\lambda_{1}>1$ arbitrarily close to $\lambda$ (from (G.A.)) and an integer $N$ such that for every $n>N$ and every $(\alpha, c) \in *(n)$ we have

$$
\min \left(\frac{\left|f^{n}(\alpha, c)\right|}{|(\alpha, c)|}, \frac{\left|f^{n}(\alpha, \beta)\right|}{|(\alpha, \beta)|}\right)>\frac{\sqrt{m}+\sqrt{M}}{\sqrt{m}} \lambda_{1}^{n} .
$$

A recollection promised in the introduction.

Definition 4. ([G, CE].) We call $p$ a central point of $f^{n}$ if $p_{n}=p$ and $\left.D f^{n}\right|_{(p, c)}>0$. We say that $p$ is restrictive if $f^{n}((c, p)) \subset(p, \hat{p})$.

$P 4$. ([N1, Proposition 4.2].) $f$ has only a finite number of iterates with a restrictive central point.

P5. ([N2, Lemma 10].) Suppose that for some $n>1, *(n)$ is nonempty. Then (a') $f^{n}$ has a central point $p$.

(b) $(\alpha, \hat{\alpha}) \subset(p, \hat{p}) \subset(\beta, \hat{\beta})$, where $(\alpha, c) \in *(n)$ and $\beta$ is associated with $\alpha$.

At the end of the next section we shall use the following facts:

LemMA 3. Suppose $(\alpha, c) \in *(n), \beta$ is associated with $\alpha$ and $p$ is a central point of $f^{n}$. If $p$ is not restrictive then there is a positive integer s such that $f^{n s}((\alpha, c)) \cap\{\beta, \hat{\beta}\} \neq \varnothing$.

Proof. Without loss of generality we may assume that $\left.D f^{n}\right|_{(\beta, c)}>0$. We have $\langle p, \hat{p}\rangle \subset$ $f^{2 n}(\alpha, c)$ as $p$ is not restrictive. We have $(p, c) \subset(\beta, c)$ and if $f^{2 n}(\alpha, c) \nexists \beta, c_{2 n} \in$ $(\beta, p)$. Let $x \in(\beta, p)$ then by $\mathrm{P} 1$ and by the facts that graph $\left(\left.f^{n}\right|_{[\beta, c]}\right)$ intersects the diagonal only at the point $p$ and that $D f^{n}(p)>1$ (both implied by (d) $\S 0$ ) we have

$$
\frac{\left|x_{n}-p_{n}\right|}{|x-p|} \geqslant \min \left(\frac{\left|c_{n}-p_{n}\right|}{|c-p|}, \frac{\left|\beta_{n}-p_{n}\right|}{|\beta-p|}\right)=\rho(n)>1 \text {. }
$$

Hence as long as $c_{(s-1) n} \in(\beta, p)$ we have

$$
\frac{\left|f^{n s}\left(c_{n}\right)-f^{n s}(p)\right|}{\left|c_{n}-p\right|}>\rho^{s}
$$

which proves the Lemma.

COROllary 1. Suppose that $f^{n}$ has no restrictive central point for $n>1$. Then there exists $m=m(N)$ such that if $(\alpha, c) \in *(n)$ for $n \leq N$ then $f^{m}((\alpha, c))=\left(c_{2}, c_{1}\right)$.

Proof. For $\beta$ associated with $\alpha$ we have $(c, \beta) \in *\left(n_{1}\right)$ for some $n_{1}<n$. Find $\beta_{1}$ associated with $\beta$, then $\beta_{2}$ associated with $\beta_{1}$ etc. The sets $f^{k}(\alpha, c)$ with growing $k$ swallow successively $\beta, \beta_{1}, \beta_{2}, \ldots$. This procedure will be described in more detail in the next section. 
Let us end this section with

P6. ([N3, Corollary 21].) There exists $\lambda_{2}>1$ such that the length of each interval of monotonicity of $f^{n}$ is smaller than $\lambda_{2}^{-n}$.

This is a deep fact which follows from (a)-(d) and (G.A.), see $\S 4$ for further discussion.

\section{Estimates}

LEMMA 4. Suppose $I=(z, y)$ be an interval such that for some $(\alpha, c) \in *(n)$ with $n>N$ we have $(\alpha, c) \subset I \subset(\beta, \hat{\beta})$, where $\beta$ is associated to $\alpha$. Then.

(a") $\left|f^{n}(I)\right| /|I|>\lambda_{1}^{n}$ and

$\left(\mathrm{b}^{\prime \prime}\right)$ either $(\alpha, c) \subset f^{n}(I)$ or $(\hat{\alpha}, c) \subset f^{n}(I)$.

Proof. Suppose first that $\left.D f^{n}\right|_{(z, y)} \neq 0$. So we assume $I=(c, y)$ and $\alpha \in I$. We estimate $\left|f^{n}(c, \alpha)\right| /|(c, \alpha)|$ with Lemma 2 , and $\left|f^{n}(\alpha, y)\right| /|(\alpha, y)|$ with $\mathrm{P} 1$, where we put $a=c$, $b=\beta ; z=\alpha ; t=y$, and Lemma 2 . We obtain

$$
\left|f^{n}(I)\right| /|I|>\lambda_{1}^{n}
$$

If $c \in(z, y)$, we call $(v, c)$ that one of two intervals $(z, c)$ and $(c, y)$ for which the length of the image under $f^{n}$ is bigger, say $v=y$. We have by $\mathrm{P}$.

$$
|(z, c)| \leq \sqrt{\frac{M}{m}}|(y, c)|=\sqrt{\frac{M}{m}}|(v, c)|
$$

and

$$
I=|(c, y)|+|(z, c)| \leq\left(1+\sqrt{\frac{M}{m}}\right)|(v, c)| .
$$

Now we use the estimates in the case $\left.D f^{n}\right|_{(c, v)} \neq 0$ and obtain

$$
\frac{\left|f^{n}(I)\right|}{|I|}=\frac{\left|f^{n}(v, c)\right|}{|I|} \geq \frac{\left|f^{n}(v, c)\right|}{\frac{\sqrt{M}+\sqrt{m}}{\sqrt{m}}|(v, c)|} \geq \frac{\sqrt{m}}{\sqrt{m}+\sqrt{M}} \cdot \frac{\left|f^{n}(v, c)\right|}{|v, c|} \geq \lambda_{1}^{n} .
$$

(b) Follows from the lack of sinks (d).

LемMA 5. There exists a constant $K_{1}>0$ such that for every $n$ if $(\alpha, c) \in *(n)$ then $|(\alpha, c)|>K_{1} \lambda_{1}^{n} L^{-2 n}$ where $L=\sup _{x \in\langle 0,1\rangle}\left|f^{\prime}(x)\right|$.

Proof. It is enough to prove the Lemma for $n>N$. By lemma 2 and P1 we have

$$
\lambda_{1}^{n}=\left|\lambda_{1}^{n}-0\right| \leq D f^{n}(\alpha)-D f^{n}(c)|\leq| \int_{\alpha}^{c}\left|\left(f^{n}\right)^{\prime \prime}(x)\right| d x \mid .
$$

We compute $\left(f^{n}\right)^{\prime \prime}$ :

$$
\left(f^{n}\right)^{\prime \prime}(x)=\left(D f^{n}(x)\right)^{\prime}=\left(\prod_{s=0}^{n-1} f^{\prime}\left(x_{s}\right)\right)^{\prime}=\sum_{i=0}^{n-1} \prod_{\substack{s=0 \\ s \neq i}}^{n-1} f^{\prime}\left(x_{s}\right) \cdot f^{\prime \prime}\left(x_{i}\right) \cdot D f^{i}(x)
$$

Thus

$$
\left|\left(f^{n}\right)^{\prime \prime}\right| \leq L^{n-1} \sup _{x \in\langle 0,1\rangle}\left|f^{\prime \prime}(x)\right| \sum_{i=0}^{n-1} L^{i} \leq K^{-1} L^{2 n} \text { for some } K>0
$$


Therefore

$$
\lambda_{1}^{n}<\left|\int_{\alpha}^{c}\right|\left(f^{n}\right)^{\prime \prime}(x)|d x| \leq K^{-1} L^{2 n}|\alpha-c|,
$$

which proves the assertion.

Remark. In fact we need not use (G.A.). We could replace $\alpha$ by a periodic point $q \in(\alpha, \hat{\alpha}), q=q_{n}$, and use the assumption that it is a source. Then we would lose $\lambda_{1}^{n}$ in the estimate. Cf, [N1, proposition 6.1].

Proposition 1. There exists $\kappa_{1}>1$ with the property that for every $n \geq 1$ there exists $m=m(n)<\kappa_{1} n$ such that if $(\alpha, c) \in *(n)$, then

$$
f^{m}((\alpha, c)) \supset\left(\alpha^{\prime}, c\right)
$$

for some $\left(\alpha^{\prime}, c\right) \in *\left(N^{\prime}\right), N^{\prime} \leq N$ ( $N$ from Lemma 2 and Corollary $1 \S 1$.) With large $N, \kappa_{1}$ can be taken arbitrarily close to $(2 \log L / \log \lambda)-1$.

Proof. Obviously for $n \leq N$ we can set $m=0$, so we assume $n=n_{0}>N$.

Let $I=(\alpha, c) \in *(n)$. We take the image under $f^{n}$ of $I, f^{n}(I), f^{2 n}(I), \ldots$, as long as $f^{k n}(I) \subset(\beta, \hat{\beta}), \quad \beta$ associated with $\alpha$. Let $s_{0}$ be such that $f^{\left(s_{0}-1\right) n}(I) \subset(\beta, \hat{\beta})$ and $f^{n s_{0}}(I) \backslash(\beta, \hat{\beta}) \neq \varnothing$. By lemma 4 such $s_{0}$ exists. Now we find an interval $\left(c, \alpha^{1}\right) \in *\left(n_{1}\right)$ for some $n_{1}<n$, such that $\left(c, \alpha^{1}\right) \subset f^{n s_{0}}(I) \subset\left(\beta^{1}, \hat{\beta}^{1}\right)$, where $\beta^{1}$ is associated with $\alpha^{1}$. Such $\alpha^{1}$ exists as at least one of two intervals $(c, \beta)$ or $(c, \hat{\beta})$ is contained in $f^{n s_{0}}(I)$. We also use the fact that $D f^{n}(\beta)=0$ as $n \geq 2$.

We repeat the whole operation and find $s_{1}, \alpha^{2}, n_{2}, \beta^{2}, s_{2}, \ldots$, until for some $j$ we have $n_{j} \leq N$. Such $j$ exists as the sequence $n_{i}$ is strictly decreasing. We have $\left(\alpha^{j}, c\right) \in *\left(n_{j}\right)$, and hence by Lemma $5\left|\left(\alpha^{j}, c\right)\right|>K_{1} \lambda_{1}^{n_{j}} L^{-2 n_{j}} \geq K_{1} \lambda_{1}^{N} L^{-2 N}$. On the other hand if we set $m=\Sigma_{0}^{j-1} n_{i} s_{i}$ we have by the construction $\left(\alpha^{j}, c\right) \subset f^{m}(I)$, and to finish the proof we have to estimate $m$. We set $I_{0}=I, I_{i+1}=f^{n_{i} s_{i}} I_{i}$ and have

$$
\frac{\left|f^{m} I\right|}{|I|}=\prod_{i=0}^{j-1} \frac{\left|f^{n_{i} s_{i}} I_{i}\right|}{\left|I_{i}\right|}=\prod_{i=0}^{j-1} \prod_{r=0}^{s_{i}-1} \frac{\left|f^{n_{i}}\left(f^{n_{i} r} I_{i}\right)\right|}{\left|f^{n_{i} r} I_{i}\right|}
$$

By construction for all $i=0, \ldots, j-1$ and $r=0, \ldots, s_{i}-1 f^{n_{i} r}\left(I_{i}\right)$ satisfies the assumptions of Lemma 4.

Thus

$$
\frac{\left|f^{m} I\right|}{|I|} \geq \prod_{i=0}^{j-1} \prod_{r=0}^{s_{i}-1} \lambda_{1}^{n_{i}}=\lambda_{1}^{\sum n_{i} s_{i}}=\lambda_{1}^{m} .
$$

We use again Lemma 5 and find that

$$
|I|=|(\alpha, c)|>K_{1} \lambda_{1}^{n} L^{-2 n}, \quad \text { as }(\alpha, c) \in *(n) .
$$

Hence

$$
1>\left|f^{m}(I)\right|>\lambda_{1}^{m}|I|>K_{1} \lambda_{1}^{m} \lambda_{1}^{n} L^{-2 n}
$$

and

$$
m<\frac{2 n \log L-n \log \lambda_{1}-\log K_{1}}{\log \lambda_{1}}
$$

which ends the proof. 
Proposition 2. There exists $\kappa_{2}$ such that for every interval $J \subset\langle 0,1\rangle$ there is $m=$ $m(J, N)<\kappa_{2} \log (1 /|J|)$ which satisfies

$$
f^{m}(J) \supset\left(\alpha^{\prime}, c\right) \in *\left(N^{\prime}\right)
$$

for some $\left(\alpha^{\prime}, c\right) \in *\left(N^{\prime}\right), N^{\prime} \leq N$.

With large $N, \kappa_{2}$ can be arbitrarily close to

$$
\frac{2 \log L-\log \lambda}{\log \lambda \log \lambda_{2}}
$$

( $\lambda_{2}$ from $\left.\mathrm{P} 6, \S 1\right)$.

Proof. By P6 for $s=\left[\log (2 /|J|) / \log \lambda_{2}\right]+1$ there exists an interval of monotonicity of $f^{s}$ contained in $J$, say $(\alpha, \beta)$, with $\alpha_{k}=\beta_{l}=c$ and $k<l<s$. We have $\left(c, \beta_{k}\right)=$ $f^{k}(\alpha, \beta) \subset f^{k}(J)$ and $\left(c, \beta_{k}\right) \in *(l-k)$. By Proposition 1 there is $m_{1}$ such that $f^{m_{1}}\left(f^{k}(J)\right) \supset\left(\alpha^{\prime}, c\right) \in *(N)$ and $m_{1}<\kappa_{1}(l-k)$. Thus we can take

$$
m(J, N)=k+m_{1}<\kappa_{1} l<\kappa_{1} s \leq \kappa_{2} \log (1 /|J|) .
$$

Proof of the Main Lemma. In view of Proposition 2 and Corollary $1, \S 1$ it is sufficient to take

$$
m=m(J, N)+m(N)<\kappa_{2} \log (1 /|J|)+m(N) .
$$

Additionally we conclude that if the $J$ 's under consideration are sufficiently small, $\kappa$ can be taken arbitrarily close to $\kappa_{2}$ i.e.

$$
\frac{2 \log L-\log \lambda}{\log \lambda \log \lambda_{2}}
$$

\section{On the conjugacy}

We shall prove in this section the theorem stated in the introduction. We note that the assumption $f^{n}$ has no restrictive central point for $n>1$ is not significant as there exists $n_{0} \geq 1$ such that $f^{n_{0}}$ has a restrictive central point $p$ and $f^{n}$ for $n>n_{0}$ has no restrictive central point, see $\mathrm{P} 4, \S 1$. We can set $F=L \circ f^{n_{0}} \circ L^{-1}$ where $L$ is a linear transformation of $(p, \hat{p})$ onto $\langle 0,1\rangle$. Then $F$ satisfies the assumptions of the Theorem.

Recall from the introduction that given $f$ satisfying the assumptions of the theorem there exists a conjugacy $h=h_{f}$ with a $\beta$-tent map $g_{\beta}, \sqrt{2}<\beta \leq 2$.

Proposition 3. There exists $\xi>0$ such that for every interval $J \subset\langle 0,1\rangle$ there is an $m<\xi \cdot \log (1 /|J|)$ which satisfies

$$
g_{\beta}^{m}(J) \supset\left(g_{\beta}^{2}\left(\frac{1}{2}\right), g_{\beta}\left(\frac{1}{2}\right)\right) .
$$

If the J's under consideration are small enough, then $\xi$ can be found arbitrarily close to $1 / \log \beta$.

One can use similar arguments as in the proof of Proposition 1, we shall, however, give a direct simple proof, as $g_{\beta}$ is a map which is very simple to cope with.

LEMMA 7. For fixed $N$ there is an $\varepsilon=\varepsilon(N)$ such that for every $J \subset\langle 0,1\rangle$ if $\left|g_{\beta}^{i}(J)\right|<\varepsilon$ for $i=0, \ldots, N-1$ then $\frac{1}{2} \in g_{\beta}^{j}(J)$ for at most one $j \in\{0,1, \ldots, N-1\}$. 
Proof. $g_{\beta}^{n}\left(\frac{1}{2}\right) \neq \frac{1}{2}$ for $n=1,2, \ldots$, as $f^{n}(c) \neq c$ for $n=1,2, \ldots$. Otherwise $f$ would have an attractive periodic orbit which contradicts (4). Let $\delta=\min _{n \leq N}\left|g_{\beta}^{n}\left(\frac{1}{2}\right)-\frac{1}{2}\right|>0$. We set $\varepsilon=\frac{1}{2} \delta \beta^{-N}$. Suppose $\frac{1}{2} \in J$ then

$$
\begin{aligned}
\min _{n \leq N} \operatorname{dist}\left(g_{\beta}^{n}(J), \frac{1}{2}\right) & \geq \min _{n \leq N} \operatorname{dist}\left(\left(g_{\beta}^{n}\left(\frac{1}{2}\right) \pm \beta^{n}|J|, \frac{1}{2}\right)\right. \\
& \geq \min _{n \leq N}\left(\left|g_{\beta}^{n}\left(\frac{1}{2}\right)-\frac{1}{2}\right|-\beta^{n}|J|\right) \\
& \geq \delta-\beta^{N} \varepsilon=\frac{1}{2} \delta>0 .
\end{aligned}
$$

If $\frac{1}{2} \in g_{\beta}^{j}(J)$ for some minimal $1<j<N$ we repeat the argument with $j \leq n \leq N$.

Proof of Proposition 3. Fix $N$ such $\beta^{N}>2 \beta_{1}^{N}$ for some $\beta_{1}>1$. Let $J \propto\langle 0,1\rangle$. Suppose that

$$
\left|g_{\beta}^{j}(J)\right|<\varepsilon(N) \text { for } j=0,1, \ldots, n-1 .
$$

We write $n=k \cdot N+s, s<N$.

$$
\begin{aligned}
\frac{\left|g_{\beta}^{n}(J)\right|}{|J|} & =\frac{\left|g_{\beta}^{s}\left(g_{\beta}^{k N}(J)\right)\right|}{|J|}=\frac{\mid g_{\beta}^{s}\left(g_{\beta}^{k N}(J) \mid\right.}{\left|g_{\beta}^{k N}(J)\right|} \prod_{j=1}^{k-1} \frac{\mid g_{\beta}^{N}\left(g_{\beta}^{j N}(J) \mid\right.}{\left|g_{\beta}^{j N}(J)\right|} \\
& \geq \frac{\beta^{s}}{2} \cdot \prod_{j=0}^{k-1}\left(\frac{\beta^{N}}{2}\right) \geq \frac{\beta_{1}^{k N+s}}{2}=\frac{\beta_{1}^{n}}{2},
\end{aligned}
$$

by Lemma 7 . Hence

$$
1>\left|g_{\beta}^{n}(J)\right| \geq \beta_{1}^{n}|J| / 2 \text { and } n \leq \frac{\log (2 /|J|)}{\log \beta_{1}} .
$$

Since $g_{\beta}$ is conjugated with $f$ we can use the topological properties of $f$, Corollary $1, \S 1$, and see that there is some integer $n_{1}=n_{1}(\varepsilon)$ such that if $\left|J^{\prime}\right| \geq \varepsilon$ for an interval $J^{\prime} \subset\langle 0,1\rangle$ then $g_{\beta}^{n_{1}}(J \mid) \supset\left(g_{\beta}^{2}\left(\frac{1}{2}\right), g_{\beta}\left(\frac{1}{2}\right)\right)$. (We could also refer to [CE, Lemma II.7.1] such that for $\beta: \sqrt{2}<\beta \leq 2, g_{\beta}$ has no restrictive central points for $n>1$.)

By (3) there exists $n$ such that (2) is satisfied and $\left|f^{n}(J)\right| \geq \varepsilon$. Hence there is an $m$ such that

$$
g_{\beta}^{m}(J) \supset\left(g_{\beta}^{2}\left(\frac{1}{2}\right), g_{\beta}\left(\frac{1}{2}\right)\right) \text { and } m \leq \frac{\log (2 /|J|)}{\log \beta_{1}}+n_{1}
$$

and the assertion follows.

Proof of Theorem. We shall prove that there are constants $\rho_{1}, \rho_{2}>0$ such that for every $x, y \in\left(c_{2}, c_{1}\right), x \neq y$

$$
\begin{aligned}
& |h(x)-h(y)| \leq|x-y|^{\rho_{1}} \\
& |x-y| \leq|h(x)-h(y)|^{\rho_{2}} .
\end{aligned}
$$

Let $m$ be a minimal number such that $f^{m}((x, y))=\left(c_{2}, c_{1}\right)$. Then $m$ is also minimal with $g_{\beta}^{m}(h(x), h(y))=\left(g_{\beta}^{2}\left(\frac{1}{2}\right), g_{\beta}\left(\frac{1}{2}\right)\right)$. By the Main Lemma and Proposition 3 we have

$$
m \leq \kappa \cdot \log (1 /|x-y|)
$$

and

$$
m \leq \xi \cdot \log (1 /|h(x)-h(y)|)
$$


On the other hand $\left|f^{m}(x, y)\right| /|(x, y)| \leq L^{m}$, where $L=\sup _{z \in\langle 0,1)}\left|f^{\prime}(z)\right|$. Thus

$$
m \geq \frac{1}{\log L} \log \left(\left|c_{1}-c_{2}\right| /|x-y|\right)
$$

and similarly

$$
m \geq \frac{1}{\log \beta} \log \left(\left|\frac{\beta^{2}-\beta}{2}\right| /|h(x)-h(y)|\right) .
$$

Now (ii) and (iii) prove (4) with $\rho_{1}=1 / \xi \log L^{\prime}$ arbitrarily close to $\log \beta / \log L$ for $x, y$ sufficiently close to each other. (This is an easy part not making use of (i) i.e. of the Main Lemma, and is true without our (G.A.).)

The inequalities (i), (iv) prove (5) with $\rho_{2}=1 / \kappa \log \beta$, for $x \approx y$, arbitrarily close to

$$
\frac{\log \lambda \log \lambda_{2}}{\log \beta(2 \log L-\log \lambda)}
$$

\section{Final remarks}

Proposition 4. Let $\left(f_{t}\right)$ be a continuous family of maps satisfying the conditions (a)-(c), $\S 0$, and $f_{t}^{\prime}(0)>1$. Then, for every parameter $t$ such that for $f_{t}$ the (G.A.), no sinks and no restrictive central point for $f_{t}^{n}, n>1$ conditions hold, we have for the respective Hölder continuity exponent $p_{2, t}$ ( see (5), § 3):

$$
\rho_{2, t} \geq K\left(\log \lambda_{1, t}\right)^{2} \text {. }
$$

Here $K>0$ is a constant which depends on the family $\left(f_{t}\right)$ but not on $t . \lambda_{1, t}$ is $\lambda$ given by (G.A.) for $f_{t}$.

If we put above $\lambda_{2}$ from P6, $\S 1$, rather than $\lambda_{1}$ this proposition would follow immediately from the approximation of $\rho_{2}$ by (6). To pass from $\lambda_{2}$ to $\lambda_{1}$ one can use the estimates of [N2].

In the case

$$
\liminf _{n \rightarrow \infty} \frac{1}{n} \log D f_{t}^{n}\left(c_{1, t}\right)=\log \lambda_{0, t}>0
$$

we can replace in (7) $\lambda_{1, l}$ by $\lambda_{0, t}$, all the replacements produce different coefficients $K$ of course $\left(c_{1, t}\right.$ is a critical value for $\left.f_{t}\right)$.

By the way we can remark that the second inequality in (G.A.) follows from the first one (with different $\lambda$ ) as does P6 (by [N2]). Putting both of them into (G.A.) was a matter of taste.

Fix $f$ satisfying the assumptions of the theorem. Let $g_{\beta}$ be the conjugated tent map. In the sequel the symbol $K$ will be used to denote various positive constant depending only on $f$. From the theorem and Lemma 5 (more precisely, Proposition $6.1,[N 1])$ we immediately obtain

$$
\left|g_{\beta}^{n}\left(\frac{1}{2}\right)-\frac{1}{2}\right| \geq K\left(\lambda_{1}^{n} L^{-2 n}\right)^{1 / \rho_{2}}
$$

for every $n \geq 1$. This implies that for the corresponding kneading sequence written in the form $A A \ldots \mathrm{AB}$ (for $A$ a finite sequence of $R$ 's and $L$ 's, $B$ an infinite sequence, 
see [CE] for the notation) the number of $A$ 's is at most $n \cdot \kappa_{3}+K$, with $\kappa_{3}$ arbitrarily close to

$$
\frac{2 \log L-\log \lambda}{\rho_{2} \log \beta}
$$

for $|\boldsymbol{A}|$ sufficiently large.

This is so because the expansion by the factor $\beta$ implies the trajectory of the point $g_{\beta}^{n}\left(\frac{1}{2}\right)$ can follow a trajectory of $\frac{1}{2}$ with the same itinerary only for a restricted time.

We can, however, derive from Proposition 1 a more precise result.

Proposition 5. Let $f$ satisfies (a)-(d) and (G.A.). Then the kneading sequence $K(f)$ is eventually strictly primary. Namely there exists $\kappa_{4}>0$ such that if $K(f)$ is written in the form

$$
(A * B) D
$$

with $|A|$ finite sufficiently large, it must be $|B|<\kappa_{4}$.

If $f$ has no restrictive central point for $n>1$, then the above is true for every $A$ of finite length $(K(f)$ is strictly primary).

Proof. Let $n=|A|+1$. Let $s$ be the smallest integer such that there exist $(\alpha, c) \in *(s)$ and $c_{n} \notin(\alpha, \hat{\alpha})$. By Proposition 6.1 [N1], $\left|c_{n}-c\right| \geq K \lambda^{n} L^{-2 n}$. By (G.A.) $|\alpha-c|<K \lambda^{-s}$. So

$$
s \leq n \cdot 2 \frac{2 \log L-\log \lambda}{\log \lambda}+K .
$$

The factor 2 before the ratio is needed as we can take into account only nonempty $*(s)$ 's. Fortunately between every $m$ and $2 m$ there is such as $s$, [N1].

Let $\beta \neq c$ be a point closest to $c$, where $D f^{n}(\beta)=0$. By Proposition 1 there exists $m<\kappa_{1} s$ such that $f^{m+\imath N^{\prime}}((\alpha, c))$ contains an interval belonging to $*\left(N^{\prime}\right), N^{\prime} \leq N$, for every non-negative integer $t$.

So for $k=[m / n]+1$ there exists $N^{\prime \prime}<N^{\prime}$ such that $f^{k n+N^{\prime \prime}}\left(\left(c, c_{n}\right)\right)$ contains an interval belonging to $*\left(N^{\prime}\right)$. This and Lemma 5 give

$$
\left|f^{k n}\left(\left(c, c_{n}\right)\right)\right| \geq K \lambda_{1}^{N_{L}-2 N_{L}-N^{\prime \prime}} .
$$

If $n$ is sufficiently large then all the intervals of monotonicity of $f^{n}$ are shorter than half of the last constant. So there exists $\beta \neq c, \beta \in f^{k n}\left(\left(c, c_{n}\right)\right)$ with $D f^{n}(\beta)=0$. Let $k$ be the smallest positive integer with this property. The end points of $f^{k n}\left(\left(c, c_{n}\right)\right)$ must then be of the form $c_{l n}, c_{r n} 2,1 \leq l, r \leq k$. As they are separated by $\beta$ the blocks of $I\left(c_{i n+1}\right)$ and $I\left(c_{r n+1}\right)$ on the first $n-1$ indices must be different from each other. So the blocks of $I\left(c_{1}\right)$ on the indices $l n+1, \ldots, l n+n-1$ and $r n+1, \ldots, r n+n-1$ must be different. The proof is finished. Let us remark that we obtained

$$
\kappa_{4} \approx\left[2 \frac{2 \log L-\log \lambda}{\log \lambda} \kappa_{1}\right]+1 \approx\left[2\left(\frac{2 \log L-\log \lambda}{\log \lambda}\right)^{2}\right]+1
$$

we got $\kappa_{4}$ similar to $\kappa_{3}$. 
Question. Is it true that for $f$ satisfying (a)-(d) with the kneading sequence eventually strictly primary, there exist an absolutely continuous, invariant, probability measure?

In $[\mathbf{J}]$ there is an example of $f$ with (a)-(d) and no restrictive central point conditions satisfied but without an a.c.i.p. measure. For this example the kneading sequence is not strictly primary.

For $f$ satisfying the assumptions of the theorem and a Hölder continuous function $\varphi$ on $\langle 0,1\rangle$ we can consider a sequence of random variables $\varphi \circ f^{n}$ on $\langle 0,1\rangle$ with the measure $m_{\max }$ of maximal entropy. Then with the use of the theorem we obtain various probability laws such as the Law of the Iterated Logarithm or Almost Sure Invariance Principle. Indeed $m_{\max }$ is the $\left(h_{f}^{-1}\right) *$ image of an absolutely continuous invariant measure $m$ for the corresponding $g_{\beta}$. The above laws for $\psi \circ g_{\beta}^{n}, \psi$ Hölder, on the probability space $(\langle 0,1\rangle, m)$ were proved in [HK].

Consider a polynomial $\mu x(1-x), 0<\mu<4$ satisfying (a) $-(d)$ and (G.A.). Extend it from $\langle 0,1\rangle$ to the Riemann sphere, $P_{\mu}(z)=\mu z(1-z)$.

CONJECTURE. The Riemann map $R$ from the unit disc $\mathbb{D}$ to the basin of attraction to $\infty$ for iteration of $P_{\mu}$ extends to a Hölder continuous map on clD.

\section{Acknowledgement}

We want to express our gratitude for the hospitality of the Mathematics Research Centre at the University of Warwick, where this paper was basically produced.

\section{REFERENCES}

[CE] P. Collet \& J-P. Eckmann. Iterated Maps on the Interval as Dynamical Systems. Birkhauser: Basel-Boston-Stuttgart, 1980.

[CE1] P. Collet \& J-P. Eckmann. Positive Lyapunov exponents and absolute continuity for maps of the interval. Ergod. Th. \& Dynam. Sys. 3 (1983), 13-46.

[CE2] P. Collet \& J-P. Eckmann. On abundance of aperiodic behaviour for maps on the interval. Comm. Math. Phys. 73 (1980), 115-160.

[C] P. Collet, Ergodic properties of some unimodal mappings of the interval. Preprint Institut Mittag-Leffler 11 (1984).

[G] J. Guckenheimer. Sensitive dependence on initial conditions for one dimensional maps. Comm. Math. Phys. 70 (1979), 133-160.

[M] M. Misiurewicz. Absolutely continuous measure for certain maps of an interval. Publ. Math. IHES 53 (1981), 17-51.

[N1] T. Nowicki. On some dynamical properties of the $S$-unimodal maps on an interval. Fund. Math. 126 (1985), 27-43.

[N2] T. Nowicki. Symmetric $S$-unimodal mappings and positive Lyapunov exponents. Ergod. Th. \& Dynam. Sys. 5 (1985) 611-616.

[N3] T. Nowicki. S-unimodal mappings and Liapunov exponents. To appear in Ergod. Th. \& Dynam. Sys.

[HK] F. Hofbauer \& G. Keller. Ergodic properties of invariant measures for piecewise monotonic transformations. Math. Zeit. 180 (1982), 119-140.

[J] S. D. Johnson. Singular measures without restrictive intervals. Comm. Math. Phys. 110 (2) (1987), 185-190. 